\title{
Epididymal Leiomyoadenomatoid Tumor: A Case Report and Review of Literature
}

\author{
Arnault Cazorla $^{a} \quad$ Marie-Paule Algros $^{\mathrm{a}} \quad$ Isabelle Bedgedjian ${ }^{\mathrm{a}}$ \\ Eric Chabannes $^{b} \quad$ Philippe Camparo $^{c}$ Séverine Valmary-Degano ${ }^{a}$
}

Department of a Pathology and bUrology, CHRU Jean Minjoz, Besançon; 'Department of Pathology, CHRU Amiens, Amiens, France

\author{
Key Words \\ Leiomyoadenomatoid tumor • Epididymis • Paratesticular • \\ Neoplasia
}

\begin{abstract}
Primary tumors of the epididymis are rare. Adenomatoid tumors are benign, usually found within the wall of fallopian tubes or beneath the uterine serosa. They are most frequently diagnosed as benign tumors of the epididymis and represent $30 \%$ of paratesticular tumors. The origin of this tumor is mesothelial cells. Leiomyoma are less common in the paratesticular localization. The origin of this tumor is smooth muscle cells. Clinically, these tumors are indistinctive with a painless mass of the scrotum. Here, we reported a case of combined leiomyoadenomatoid tumor. The histogenesis of this lesion remains unknown. This entity can be the result of a collision of the two tumors, or it can be a subtype of adenomatoid tumors with smooth muscle hyperplasia. This case showed the difficulty that occurs in the identification of this kind of lesion. Only one case of this entity in the epididymis was described in the literature. Leiomyoadenomatoid tumor is a benign neoplasm. In our case, this lesion was surgically removed in toto and no recurrence was observed.
\end{abstract}

Copyright ๑ 2013 S. Karger AG, Basel

\section{Introduction}

Tumors of the epididymis are rare and mainly benign. Adenomatoid tumors are benign neoplasms of mesothelial origin that occur most frequently in the epididymis

\section{KARGER}

Fax +4161306 1234

E-Mail karger@karger.ch

www.karger.com
(C) 2013 S. Karger AG, Basel

1015-9770/13/0074-0195\$38.00/0

Accessible online at:

www.karger.com/cur in males and in the myometrium or fallopian tubes in females [1]. They constitute the most common tumor of the epididymis, and are usually encountered in adults and in the elderly [1]. Epididymal leiomyoma is the second most common tumor of the epididymis and is also seen in adults [2]. When there is a prominent smooth muscle component within the adenomatoid tumor, the lesion is denoted as "leiomyoadenomatoid tumor". Here we report a case of a leiomyoadenomatoid tumor in a 57-yearold man.

\section{Case Report}

A previously healthy 57-year-old man, with a personal history of right orchiepididymitis one year before, was referred to an urologist for a chronic painless intrascrotal mass. The lesion had been present for several years and had not increased in size. Physical examination revealed a mobile, firm, painless mass $2 \mathrm{~cm}$ in diameter in the right scrotum. The left testis was of normal size and consistency. Nothing relevant was found in the rest of the physical examination. Routine laboratory tests including complete blood count, blood biochemistry, serum $\alpha$-fetoprotein, placental alkaline phosphatise, and $\beta$-human chorionic gonadotrophin levels were within normal limits. Scrotal ultrasonography revealed a solid, extratesticular, well-limited, heterogeneous, and mainly hypoechogenic mass of $15 \times 20 \mathrm{~mm}$ (fig. 1). Subsequently, right inguinal exploration was performed, during which a firm solid mass arising from the tail of the epididymis was found. Fresh frozen sections of the tumor were diagnosed as a benign tumor with spindle-shaped cells of the epididymis. The tumor was excised in toto with the vaginalis tunical after high ligation and transection of the spermatic cord. The testis was normal. The patient's postoperative course was uneventful.

On gross examination, the tumor was a firm encapsulated mass with a fasciculated aspect. The mass measured $25 \mathrm{~mm}$ in diameter. There was a large central area of coagulation necrosis (fig. 2). 


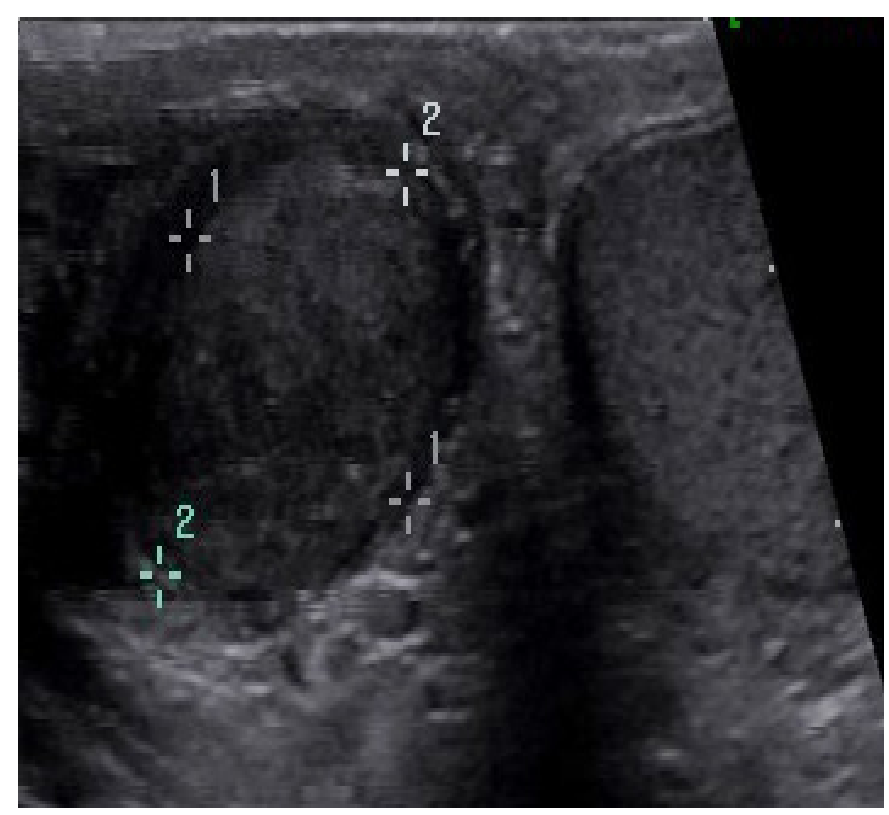

Fig. 1. Scrotal ultrasonography: a solid, extratesticular, well-limited, heterogeneous, and mainly hypoechogenic mass of 15 x 20 $\mathrm{mm}$.

Microscopically, the tumor was defined by two components. The first consisted of some gland-like spaces lined by flat or cuboidal cells with monomorphic nuclei and cytoplasmic vacuoles (fig. 3a). These epithelioid structures in the periphery were separated by a second aspect composed of fibromuscular proliferation which made up the majority of the tumor (fig. 3d). There was little nuclear atypia and mitotic activity. Immunohistochemical analysis showed epithelial-like tumor cells stained by cytokeratin 7 and calretinin (fig. $3 \mathrm{~b}$ and c). These cells did not show a positive reaction for carcinoembryonic antigen. Fascicles of the spindle cells showed a positive reaction for desmin and smooth muscle actin (fig. $3 \mathrm{e}$ and $3 \mathrm{f}$ ).

The two components observed, mesothelial and muscular, permitted the diagnosis of a leiomyoadenomatoid tumor. After 1 year, our patient was asymptomatic with no recurrence of the tumor.

\section{Discussion}

Epididymal adenomatoid tumor, originating from mesothelial cells, represents $30 \%$ of all paratesticular tumors and constitutes $65 \%$ of the total of benign neoplasms that develop in these zones $[1,3]$. In males, this lesion can occur at any time throughout life, but is is more common between 30 and 50 years-old [3]. Leiomyomas are benign tumors originating from smooth muscular cells.

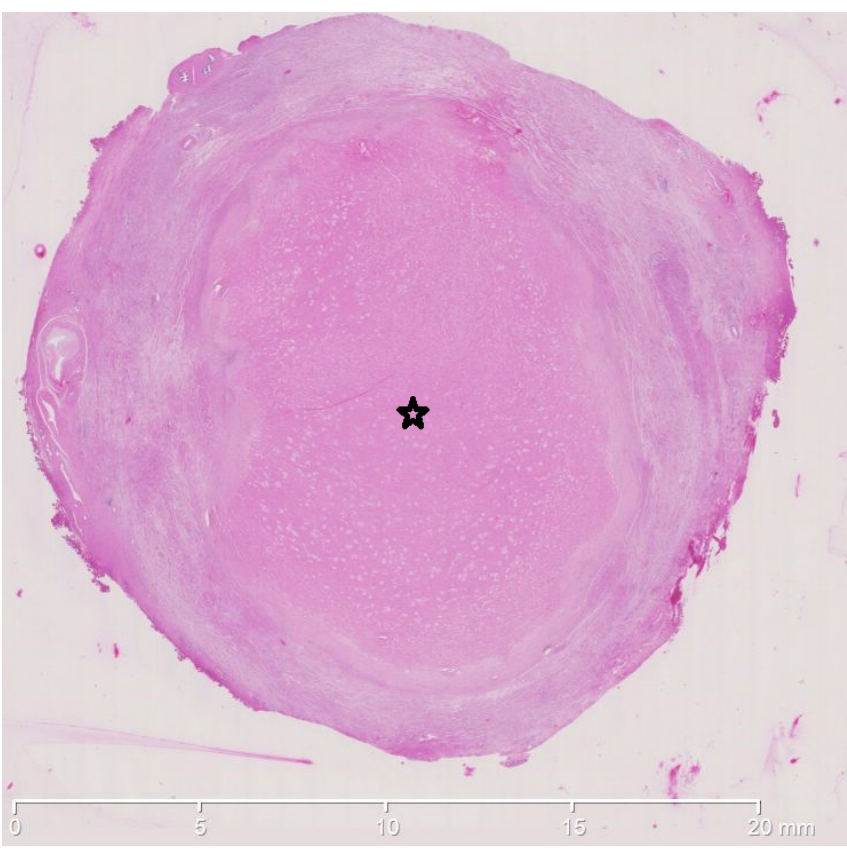

Fig. 2. Microscopic findings: an encapsulated, fasciculated tumor with a large central area of coagulation necrosis (asterisk) (HES, magnification $\times 3$ ).

In males, although patient age varies widely, this tumor most commonly manifests in the fifth decade of life as a slow-growing painless scrotal mass [2]. The term of leiomyoadenomatoid tumor was first used by Epstein, to describe a variant of adenomatoid tumor with a prominent smooth muscle component [4]. Sometimes, the smooth muscle overgrowth is so extensive that it obscures the adenomatoid tumor, and results in misdiagnosis of leiomyoma or malignant tumor infiltrating smooth muscle bundles [5]. Nevertheless, there is very little smooth muscle in the epididymis. In the literature, 5 cases of leiomyoadenomatoid tumor have been reported: one in the epididymis and the others in the uterine wall, with one of them also affecting an ovary [5-9]. Adenomatoid tumors often affect the inferior pole and rigth side of the testicle, such as in the only case of the literature and in our case [8]. The histogenesis of leiomyoadenomatoid tumors is subject to debate [3, 5-7]. Some authors support the hypothesis that this rare entity should be considered as a subtype of adenomatoid tumors, others think that it represents a collision neoplasia, and others think that it is the result of a common adenomatoid tumor associated to a reactive smooth muscle hyperplasia $[3,5]$. 

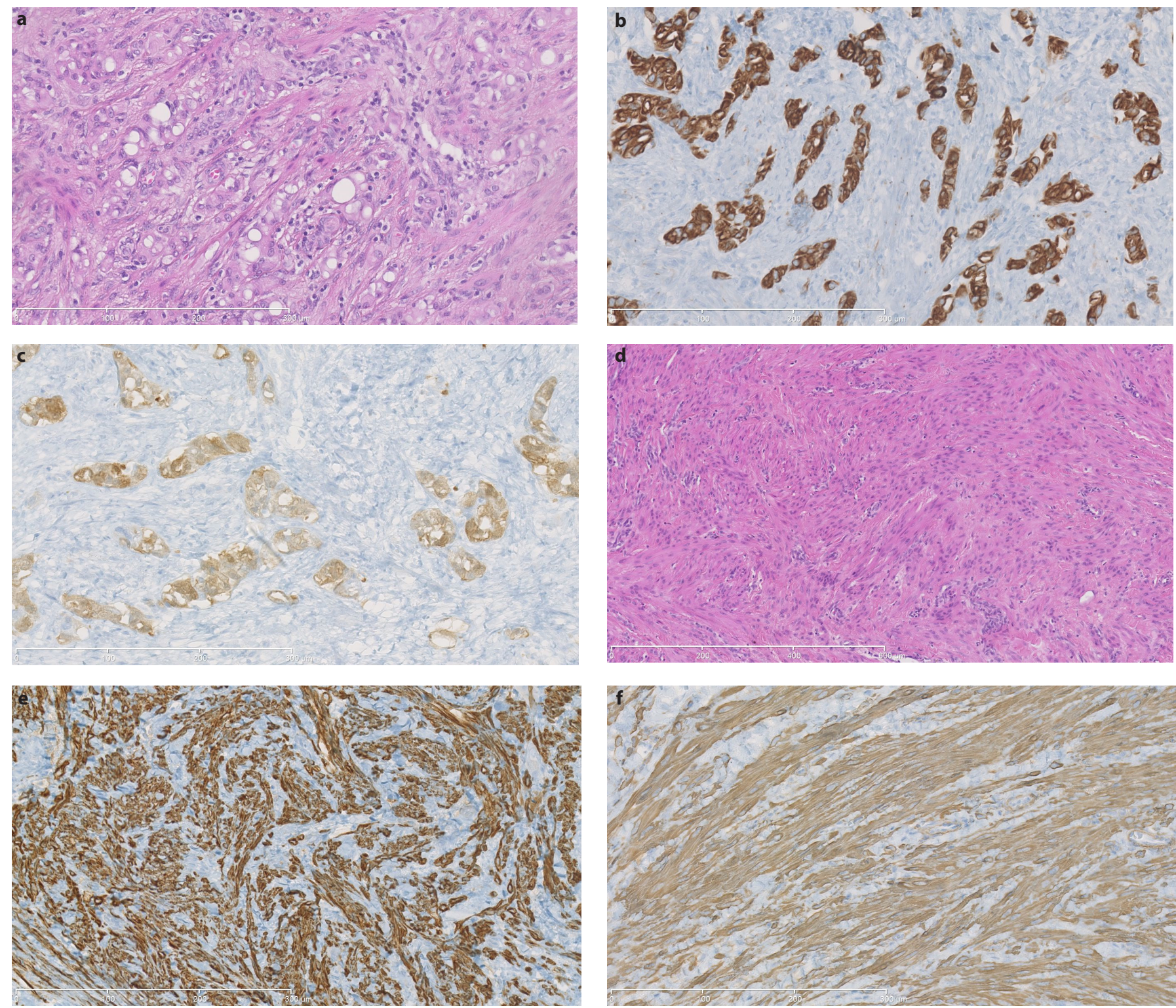

Fig. 3. Morphological and immunohistochemical findings: the gland-like component (a, b, c) with flat or cuboidal cells and monomorphic nuclei and cytoplasmic vacuoles (a HES, magnification $\mathrm{x}$ 100) express cytokeratins (b KL1 antibody, magnification x 200) and calretinin (c magnification x 200). The fibromuscular component (d, e, f) with fascicles of spindle cells (d HES, magnification x 100) showed expression of desmin (e magnification $\mathrm{x} 200$ ); and smooth muscle actin (f magnification x200).

The present case shows a well-defined neoplastic process with a biphasic pattern: an adenomatoid tumoral component intermixed with fascicles of smooth muscle cells. These criteria favor the hypothesis that a leiomyoadenomatoid tumor should be considered as a variant of an adenomatoid tumor that originated in pre- cursor cells with dual differentiation, mesothelial and muscle cells. Moreover, the location of this tumor in our case, the epididymis, which is an anatomic site with very few or absent muscular tissue, supports this hypothesis [3]. The discrepancy between the interpretation of fresh frozen and final routinely processed tumor samples in 
our case reflects the difficulty of appropriate tissue sampling. It has been postulated that inflammation may have some role in the development of adenomatoid tumors [3]. Our patient has the background of homolateral orchi-epididymitis. Clinically, epididymal leiomyomas and adenomatoid tumors present as solid, firm, limited and painless masses that are small and very slow growing [8]. The other case of epididymal leiomyoadenomatoid tumor found in the literature and our patient presented all of these characteristics. Blood studies, including tumor markers ( $\alpha$-fetoprotein, $\beta$-human chorionic gonadotrophin, and placental alkaline phosphatase) revealed normal parameters [8]. Ultrasonographic examinations are a diagnostic tool that excludes malignant lesions [8]. In the literature, the only case of an epididymal leiomyoadenomatoid tumor was heterogeneous, hypo- and hypererechogenic, extratesticular, and well-defined [8]. Doppler ultrasound demonstrated enhanced signals [8]. Those characteristics were also present in our case.

On gross examination, leiomyoadenomatoid tumors are described as pale nodules with well-circumscribed borders and whorled cut surfaces, with hard consistency [5-9]. Microscopically, the tumor consists of two components. There are smooth muscle spindle cells arranged in interlacing bundles, positive for desmin and smooth muscle actin [5-9]. The adenomatoid component has histological characteristics of 2 structures: epithelial cells and fibrous stroma. The epithelial cells are cuboidal and flat, with vacuolated cytoplasm which forms laces or glandular structures. The stroma is variable in its density [5-9]. These epithelial cells express calretinin and the epithelial membrane antigen and are negative for expression of other epithelial and endothelial markers [5-9]. One case in the literature presented multicentric infarcted aspects [5]. In our case, there was a central area of coagulation necrosis. The cause of the infarction remains unclear. This necrosis can induce confusion in the diagnosis of benignity. The adenomatoid component is not often the dominant component and careful sampling of the specimen is necessary to highlight it and allow the diagnosis.

No recurrence or malignant degeneration has ever been reported [5-9]. Nevertheless, all the authors recommend the excision of adenomatoid tumors, as was performed in our patient [5-9].

In summary, we have described a case of an epididymal leiomyoadenomatoid tumor. This entity, diagnosed with the coexistence of two components, adenomatoid and smooth muscle, is extremely rare and has to be known by pathologists.

\section{Acknowledgments}

The authors thank Sarah Clerc and Jean-Baptiste Aupet for their help in editing the manuscript.

\section{References}

1 Alvarez Maestro M, Tur Gonzalez R, Alonso Dorrego JM, Jesus De la Pena Barthel J, Nistal Martin de Serrano M: Adenomatoid tumors of the epididymis and testicle: report of 9 cases and bibliographic reviews. Arch Esp Urol 2009;62:137-141.

2 Ozden O, Orhan D, Karnak I: Epididymal leiomyoma: an unusual intrascortal tumor in a child. J Pediatr Surg 2009;44:E5-7.

-3 Pila Perez R, Rosales Torres P, Pila Pelaez R, Holguin Prieto V, Torres Vargas E: Adenomatoid tumor of the epididymis: an infrequent case. Arch Esp Urol 2009;62:656-660.

- Epstein JI: Differential diagnosis in pathol-

4 ogy: urologic disorders. Igaku-Shoin, New York, 1992, pp173-174.
5 Hong R, Choi DY, Choi SJ, Lim SC: Multicentric infracted leiomyoadenomatoid tumor: a case report. Int J Clin Exp Pathol 2009;2: 99-103.

6 Amre R, Constantino J, Lu S, Charney D: Pathologic quiz case. A 52-year-old woman with a uterine mass. Arch Pathol Lab Med 2005;129:77-78.

-7 Erra S, Pastormerlo M, Gregori G, Costamagna D, Pavesi M: A case of leiomyoadenomatoid tumour of uterine serosa: speculations about differential diagnosis. BMJ Case Rep 2009;2009:bcr02.2009.1586.
8 Kausch I, Galle J, Buttner H, Bohle A, Jocham D: Leiomyo-adenomatoid tumor of the epididymis. J Urol 2002;168:636.

-9 Amérigo J, Amérigo-Góngora M, GiménezPizarro A, Velasco FJ, Gallardo SP, González-Cámpora R: Leiomyo-adenomatoid tumor of the uterus: a distinct morphological entity? Arch Gynecol Obstet 2010;282:451-454. 